\title{
Simulations of transonic flow fields around an elastic arrow wing
}

\author{
Eddie Ly* John A. Gear* Masato Tamayama ${ }^{\dagger}$
}

(Received 10 October 2005; revised 10 August 2006)

\begin{abstract}
Transonic flows are characterised by the presence of adjacent regions of subsonic and supersonic flow, usually with shock waves present at the downstream supersonic-subsonic interface. An in-house FORTRAN90 computer code, TRANFLOW3D, which is robust and capable of simulating complex nonlinear flow fields around a double-swept-back semispan supersonic transport wing model (of high sweep angle) at transonic speeds is presented. The code has acceptable turnaround times on current high performance personal computers. The flow is governed by the general frequency, nonlinear, transonic small disturbance equation, subject to nonreflecting, far field, boundary conditions. Numerical solution procedure based on finite difference method,
\end{abstract}

*School of Mathematical \& Geospatial Sciences, Science, Engineering \& Technology Portfolio, RMIT University, Melbourne, Australia. mailto: eddie.ly@rmit.edu.au

$\dagger^{\dagger}$ Aeroelasticity Section, Structure Technology Center, Japan Aerospace Exploration Agency, Tokyo 182-8522, JAPAN. mailto:masato@chofu.jaxa.jp

See http://anziamj .austms.org.au/V47EMAC2005/Ly for this article, (c) Austral. Mathematical Soc. 2006. Published October 3, 2006. ISSN 1446-8735 
method of false transients and approximate factorisation technique is utilised. Chordwise distributions of steady pressure coefficient and local Mach numbers are computed, and compared with those obtained from wind tunnel measurements.

\section{Contents}

1 Introduction

2 Experimental simulation

3 Numerical simulation

C314

3.1 Governing equation and boundary conditions . . . . . . . .

3.2 Algebraic mapping process . . . . . . . . . . . . . .

C315

3.3 Numerical solution procedure . . . . . . . . . . .

4 Comparison and discussion of results

5 Concluding remarks

C321

References

C322

\section{Introduction}

In Japan, a national project on supersonic transport (SST) aircraft has started, which includes flight experiments with scaled SST models, with the objectives of establishing Japan's own technologies in design techniques using computational fluid dynamics (CFD) and to accumulate verification data [10] for in-house developed aeroelasticity and CFD related computer codes. TRANFLOw3D, which is a type dependent finite difference method based upon inhouse computer code implementing the method of false transients and the ap- 
proximate factorisation (AF) technique, solves the three-dimensional, general frequency, nonlinear transonic small disturbance (TSD) equation $[3,5,7,8]$. The code is used to generate reliable approximate results for high-sweptangle wings, like the double-swept-back SST arrow wing modelled in this paper. Although, the TSD equation is one of the simplest forms of nonlinear aerodynamics, the equation is capable of accurately predicting the strength and location of weak shock waves, and because of its efficiency (relatively low computational cost and simplicity of the griding and geometry preprocessing), is generally appropriate for simulation of transonic flows.

Modification to the inviscid TSD theory and TRANFLOW3D code is made to include the shock generated entropy and vorticity effects [7, 8, 12], so that flow fields with strong embedded shock waves can be correctly captured. In the closure, comparison of computed results with JAXA's (Japan Aerospace Exploration Agency) experimental data [10] will be made and discussed.

\section{Experimental simulation}

The model considered in the simulation is an elastic, semispan, double-sweptback arrow wing with a fuselage, as shown in Figure 1 including relevant wing specifications. The wing surface is constructed of aluminium alloy and urethane resin, and has an inboard trailing-edge flap of about $9.6 \%$ chord between $20 \%$ and $50 \%$ semispan, which can oscillate harmonically to produce unsteady flow fields around the wing. Tamayama conducted the experiments using the closed, continuous and pressurised/depressurised circulatory transonic wind tunnel at JAXA in Tokyo, Japan, for free stream Mach numbers between 0.8 and 0.95 , and at different incidence and flap angles. 


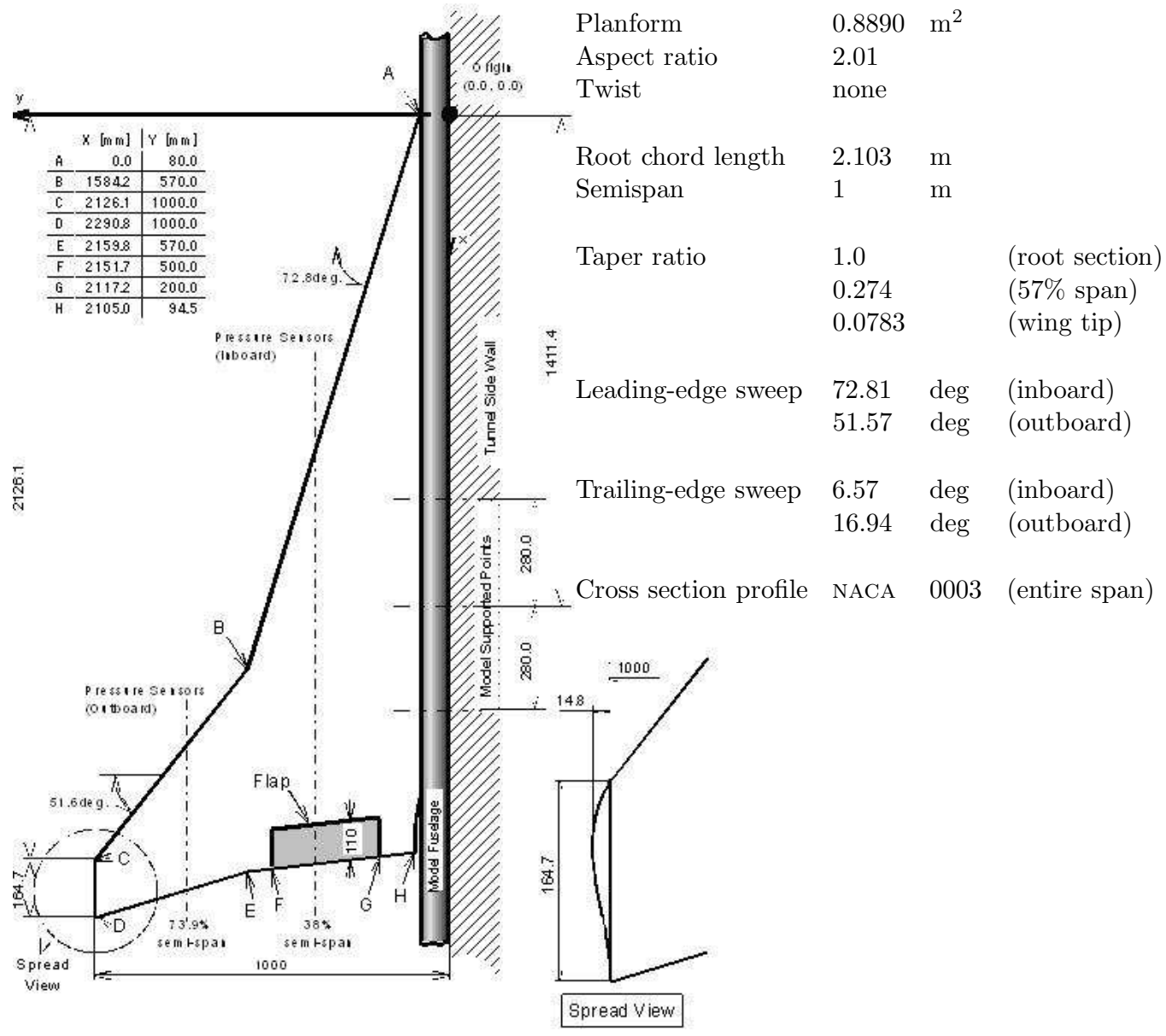

FiguRE 1: JAXA's scaled SST model. 


\section{$3 \quad$ Numerical simulation}

\subsection{Governing equation and boundary conditions}

A nearly planar wing is immersed in an unsteady, isentropic, and inviscid flow. The governing aerodynamic equation of motion is the TSD equation [1, $3,5,7,8]$, which for the reduced potential $\phi(x, y, z, t)$ is

$$
M_{\infty}^{2} \frac{\partial}{\partial t}\left[\phi_{t}+2 \phi_{x}\right]=\frac{\partial}{\partial x}\left[F\left(\phi_{x}-\bar{u}\right)^{2}+G \phi_{y}^{2}\right]+\frac{\partial}{\partial y}\left[\left(1+H \phi_{x}\right) \phi_{y}\right]+\frac{\partial}{\partial z} \phi_{z} ;
$$

where $M_{\infty}$ is the free stream Mach number, $\beta^{2}=1-M_{\infty}^{2}, \bar{u}=-\beta^{2} /(2 F)$ (denotes the value of $\phi_{x}$ at sonic condition), and $\phi_{x}=\partial \phi / \partial x$, etc. The spatial coordinates $(x, y, z), t$ and $\phi$ have been nondimensionalised by $\ell$, and $\ell / U_{\infty}$, and $\ell U_{\infty}$, where $\ell$ and $U_{\infty}$ are the aerofoil chord length measured at wing root section and free stream fluid speed, respectively. In nondimensional terms, the fluid velocity vector is $\boldsymbol{v}=\nabla(x+\phi)$. Equation (1) captures nonlinear flow phenomena, including the irregular shock wave motion observed experimentally by Tijdeman [11]. Equation (1) is locally of elliptic/hyperbolic type representing subsonic/supersonic flow when $\phi_{x}$ is less/greater than $\bar{u}$, and the solution contains discontinuous jumps that approximate shock waves. Several choices are available within TRANFLOW3D for the coefficients $F, G$ and $H$, as tabulated in Table 1, depending upon the assumptions used in deriving the TSD equation, and $\gamma$ represents the ratio of specific heats, about 1.4 for ambient air. The linear equation (where $F, G$ and $H$ are all zero) also known as the subsonic/supersonic small disturbance equation when $M_{\infty}$ is less/greater than one.

In the flow field, nonreflecting boundary conditions derived from the theory of wave propagation are utilised at the outer boundaries of the computational region, and the Kutta condition is satisfied in the wake region behind the wing $[1,3,5]$. TRANFLOW3D offers two different sets of nonreflecting boundary conditions for use in the simulations; one is based on the work of 
TABLE 1: Formulae for coefficients $F, G$ and $H$.

\begin{tabular}{|lccc|}
\hline Coefficients Type & $F$ & $G$ & $H$ \\
\hline NASA Ames & $-\frac{1}{2}(\gamma+1) M_{\infty}^{2}$ & $\frac{1}{2}(\gamma-3) M_{\infty}^{2}$ & $(1-\gamma) M_{\infty}^{2}$ \\
NLR & $-\frac{1}{2}\left[3+(\gamma-2) M_{\infty}^{2}\right] M_{\infty}^{2}$ & $-\frac{1}{2} M_{\infty}^{2}$ & $-M_{\infty}^{2}$ \\
Classic equation & $-\frac{1}{2}(\gamma+1) M_{\infty}^{2}$ & 0 & 0 \\
Linear equation & 0 & 0 & 0 \\
\hline
\end{tabular}

Gear [3, 5], and the other due to Batina [1]. Furthermore, smoothing of the wing slopes is sometimes required for highly swept wings, so that unrealistic results (high pressure peaks) at the leading edge will not form as part of the solution. In the smoothing process, the slope is altered in a smooth continuous manner at all points along the wing profile, so that when the slope is very large (near the leading edge) the slope reduces to unity. Elsewhere, when the slope is small, little change occurs. Such modifications are applied locally to enhance stability without degrading the results outside of the nose region.

\section{$3.2 \quad$ Algebraic mapping process}

To facilitate the use of high density of grid points surrounding the wing $[3,5]$, without resolving to utilising a large number of points, a smooth nonuniform computational mesh is constructed based on the following mapping functions,

$$
\xi=\xi(x, y), \quad \eta=\eta(y) \text { and } \quad \zeta=\zeta(z),
$$

where $(\xi, \eta, \zeta)$ are the dimensionless computational coordinates in the $(x, y, z)$ direction. The far field boundaries are kept independent of the wing and aligned with respect to the free stream direction, so that both the physical and computational domains are contained within rectangular block regions. Figure 2 depicts a transformed grid (with most grid planes removed for clarity in the illustration) used in the simulations, it consists of 90, 45 and 100 

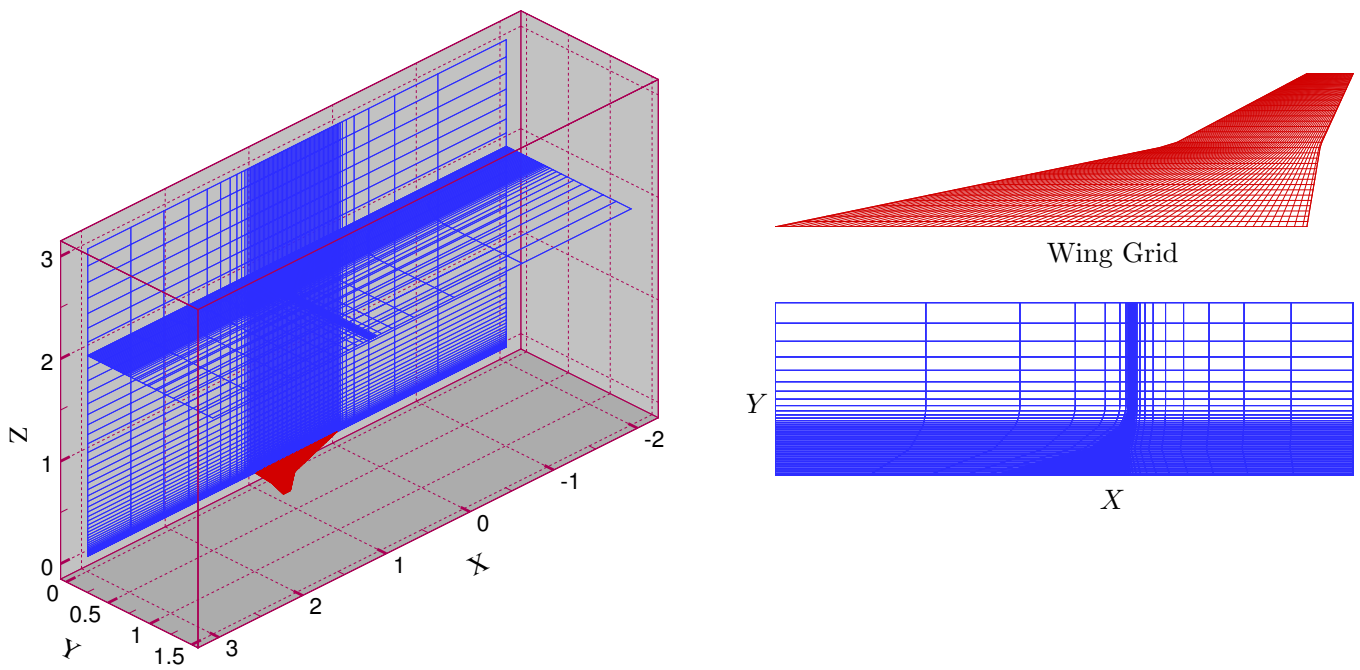

$X$

FiguRE 2: Transformed grid used in the simulations.

points in the $(\xi, \eta, \zeta)$ directions, a total of 405,000 internal grid points. On the wing, 64 and 30 points are allocated in the chordwise and spanwise direction, respectively, with 10 and 16 points for the region upstream and downstream (wake region) of the wing, and 15 points for the region extended between the wing tip and far field spanwise boundary. The far field streamwise computational boundaries are positioned at a distance of $2 \ell$ from the wing, the spanwise boundary at $1.5 \ell$ from the wing symmetry axis, and vertical boundaries are at $3 \ell$ above and below the wing.

\subsection{Numerical solution procedure}

The method of false transients $[4,6]$ is employed to compute steady-state solutions, where the time derivative of the TSD equation, Equation (1), is replaced by an "artificial" time derivative to incorporate temporal numerical dissipation. This term is then approximated by a generalised time difference 
rule, written in Padé form at time-level $\tau_{n}=n \Delta \tau$, resulting in

$$
\begin{aligned}
\frac{\partial}{\partial \tau} \frac{\phi^{n}}{J}= & \frac{\partial}{\partial \xi}\left[\frac{\xi_{x}}{J} F\left(\xi_{x} \phi_{\xi}^{n}-\bar{u}\right)^{2}+\frac{\xi_{x}}{J} G\left(\xi_{y} \phi_{\xi}^{n}+\eta_{y} \phi_{\eta}^{n}\right)^{2}\right. \\
& \left.+\frac{\xi_{y}}{J}\left(1+H \xi_{x} \phi_{\xi}^{n}\right)\left(\xi_{y} \phi_{\xi}^{n}+\eta_{y} \phi_{\eta}^{n}\right)\right] \\
& +\frac{\partial}{\partial \eta}\left[\frac{\eta_{y}}{J}\left(1+H \xi_{x} \phi_{\xi}^{n}\right)\left(\xi_{y} \phi_{\xi}^{n}+\eta_{y} \phi_{\eta}^{n}\right)\right]+\frac{\partial}{\partial \zeta}\left[\frac{\zeta_{z}}{J} \zeta_{z} \phi_{\zeta}^{n}\right] .
\end{aligned}
$$

Here $\tau$ is the artificial time scale, $\Delta \tau$ is the artificial time step, $J$ is the Jacobian of the transformation matrix, and $\phi_{\xi}^{n}$ denotes the value of $\phi_{\xi}$ evaluated at time level $\tau_{n}$.

Equation (2) is approximately factorised [6], and second order accurate central difference rules are then applied to all spatial derivatives; except for the first nonlinear spatial term which is evaluated by second order accurate Engquist-Osher type dependent operators in either standard form due to Engquist and Osher [2], or the modified form developed by Phillips, Gear and Ly $[3,9]$. Conservative differencing of the governing equation is preserved, an essential requirement for a proper description of the shock waves. The resulting scheme is potentially fast, because the solution process is fully vectorised and time-step cycling is used to enhance the rate of convergence.

Rotational effects become significant when strong shock waves exist in the flow field, since vorticity is generated due to the entropy changes along the shock. Such effects are excluded in the conventional TSD theory because of the irrotationality assumption necessary for the existence of a velocity potential. In this article, the streamwise flux of Equation (2) is modified to accommodate such effects by treating the velocity vector as a sum of potential and rotational components [7, 8, 12], and the rotational component assumed to exist only in the region behind the shock wave. 

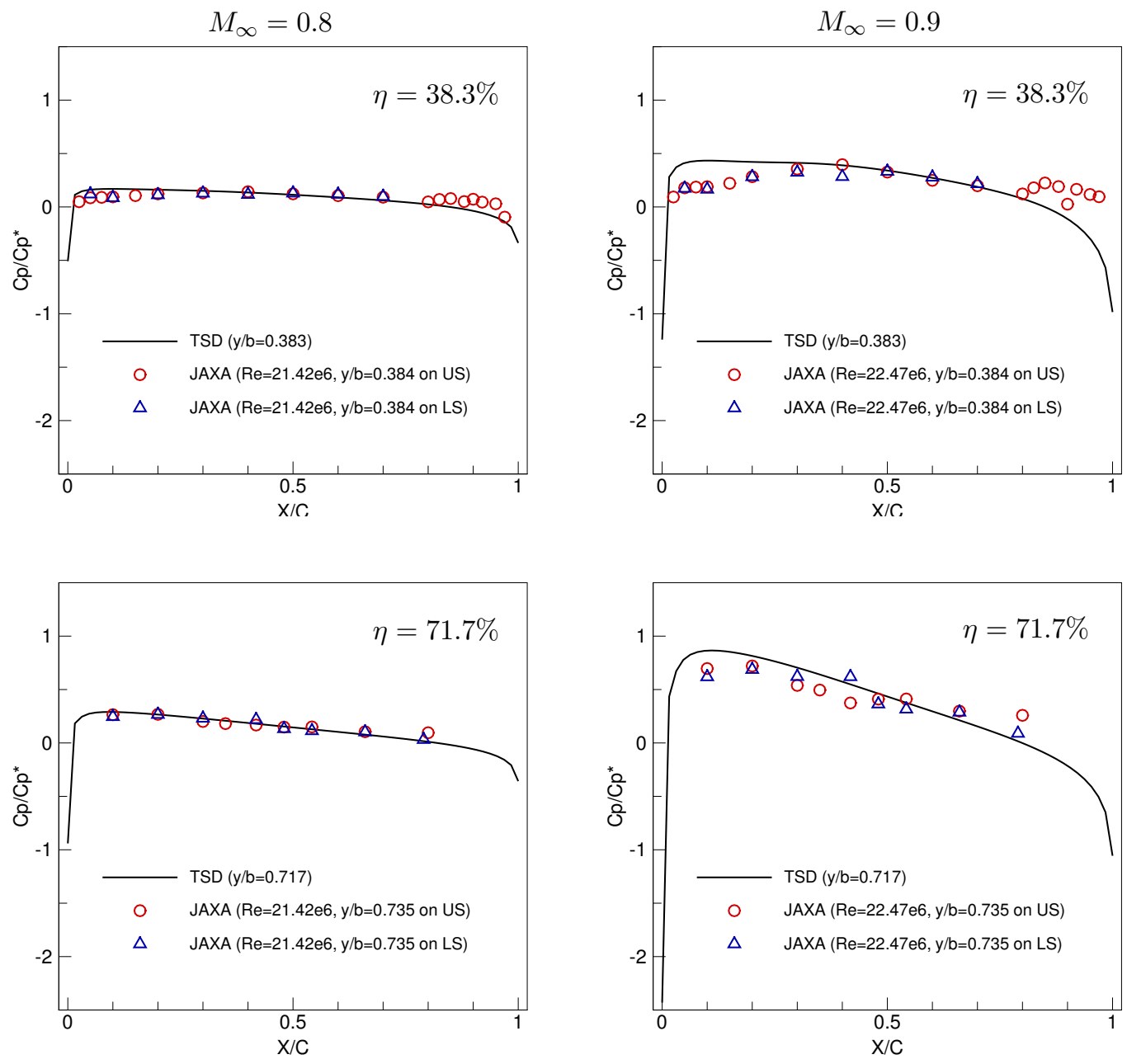

Figure 3: Comparison of results $\left(\alpha=0^{\circ}, \delta=0^{\circ}\right)$. 

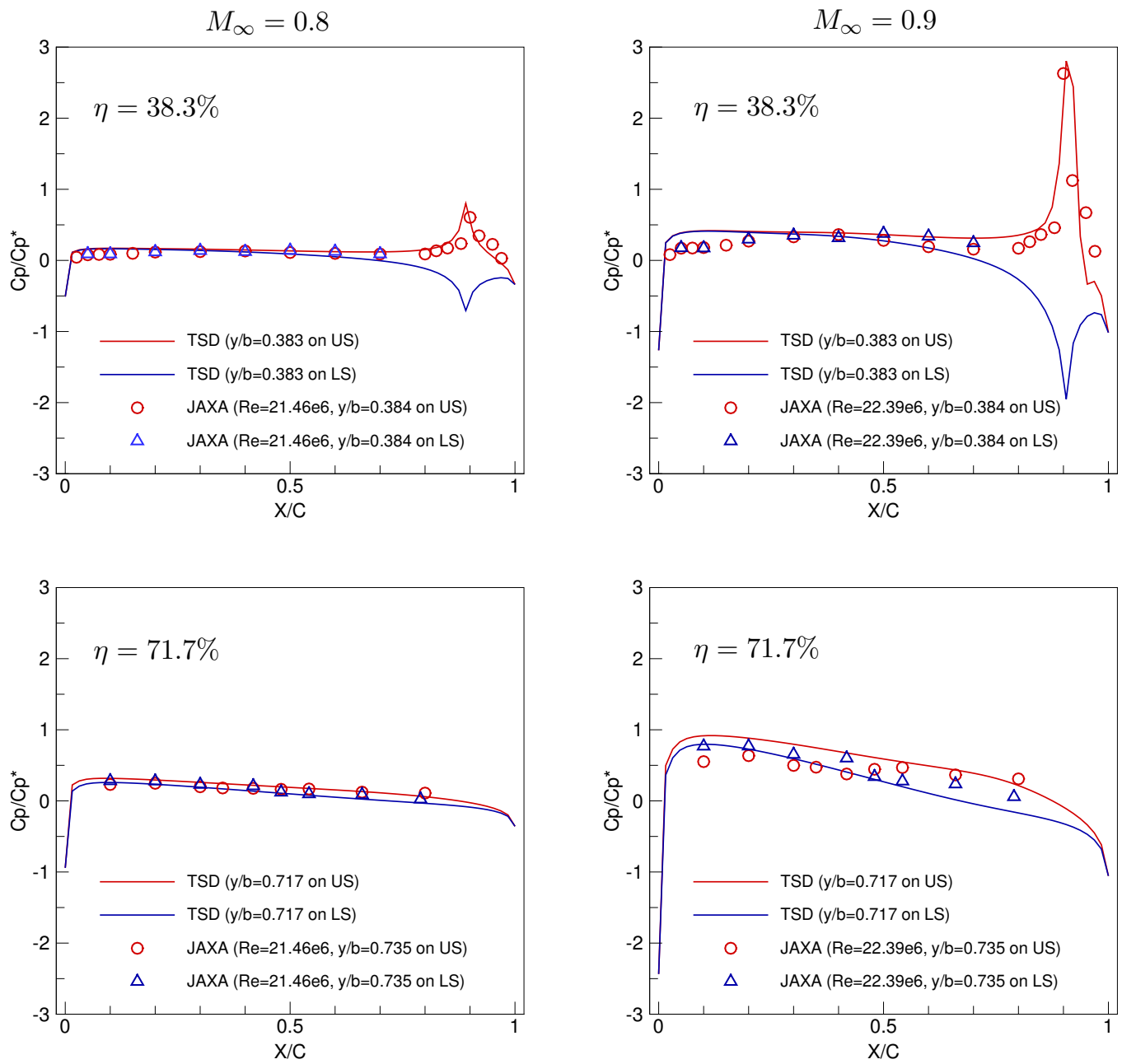

Figure 4: Comparison of results $\left(\alpha=0^{\circ}, \delta=5^{\circ}\right)$. 


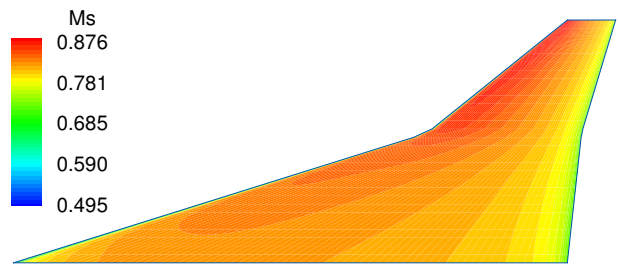

$M_{\infty}=0.8$

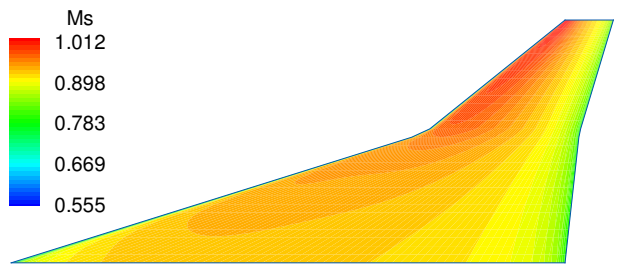

$M_{\infty}=0.9$

Figure 5: Steady local Mach numbers $\left(\alpha=0^{\circ}, \delta=0^{\circ}\right)$.

\section{Comparison and discussion of results}

Once the reduced potentials are determined, pressure coefficients, $C_{p}(x, y, z, t)$, are computed using a first order approximation formula, $C_{p}=-2 \phi_{x}$. A second order formula, $C_{p}=-\left(2+\beta^{2} \phi_{x}\right) \phi_{x}$, is also available for use within the code.

Numerical simulations were performed for the wing model at angle of attack $\alpha=0^{\circ}$ with the inboard flap deployed at $\delta=0^{\circ}$ and $\delta=5^{\circ}$ angle, and compared with the JAXA's experimental data [10] in Figures 3 and 4 . In these figures, $C_{p}$ is scaled with the critical pressure coefficient, $C_{p}^{*}=-2 \bar{u}$, and so locally supersonic points are indicated by $C_{p} / C_{p}^{*}>1$ values. The computed results compare very well with the experimental data, especially the well-captured pressure peaks due to the flap as shown in Figure 4, except for some discrepancies which exist in the flap region. This may be caused by the manner in which the wing model is constructed. In the outboard wing, all pipes and electrical wires from the pressure transducers were drawn to the inboard wing passing through a channel on the lower wing surface, and filled with resin where the finishing of the resin surface might be inadequate. A small supersonic region in an otherwise subsonic flow field begins to develop in the outboard region of the wing at $M_{\infty}=0.9$ (see Figure 4) and a shock wave begins to develop in the nose region near the wing tip as shown in Figure 5. In Figure 6 the shock wave becomes fully developed when $M_{\infty}$ reaches 0.95 , 


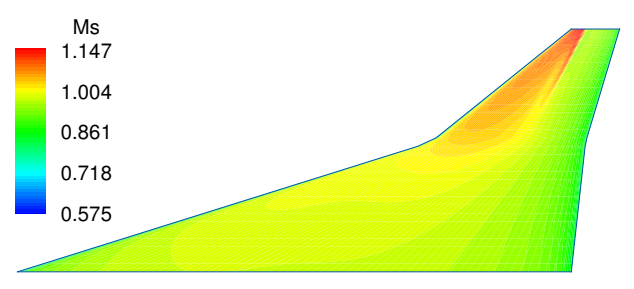

$M_{\infty}=0.95$

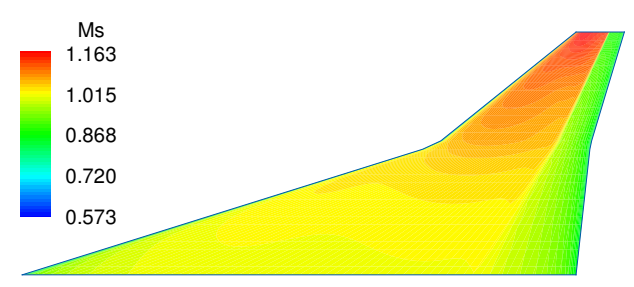

$$
M_{\infty}=0.98
$$

Figure 6: Steady local Mach numbers $\left(\alpha=0^{\circ}, \delta=0^{\circ}\right)$.

and moves from about $25 \%$ to $75 \%$ chord as $M_{\infty}$ increases from 0.95 to 0.98 . Figure 7 depicts the steady local Mach numbers distribution on the wing upper and lower sides at $M_{\infty}=0.98$ with the flap deployed at $5^{\circ}$ angle. Shock waves are clearly shown on both sides of the wing, with the upper side shock advanced further towards the trailing edge than the lower side counterpart. Also, a high pressure region due to the flap is evident.

\section{Concluding remarks}

The solution method, incorporating the wing slope smoothing and shock generated entropy and vorticity effects, has been developed and applied to the JAXA's elastic SST wing model. Excellent comparison between the computed results and wind tunnel measurements indicated that realistic accurate transonic flow solutions can be obtained from using the TRANFLOW3D code. TRANFLOW3D is an ideal tool that allows researchers to perform threedimensional transonic aeroelastic analysis (for which only small incidences are considered), and for students to experience aerodynamic computations, in acceptable turnaround times on current high performance personal computers. Finally, there is a future potential for a three-dimensional time linearised version (for which only infinitesimal amplitude motions need be considered) as a fast method to be used for unsteady flutter prediction. 

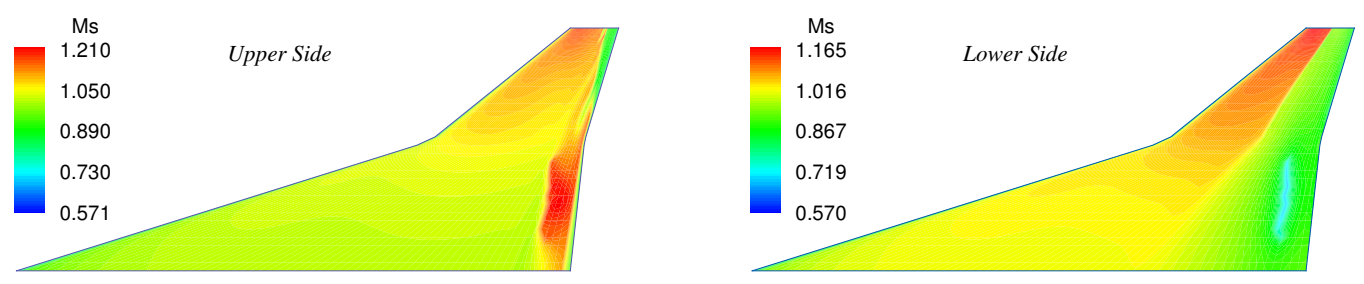

Figure 7: Steady local Mach numbers $\left(\alpha=0^{\circ}, \delta=5^{\circ}\right)$.

\section{References}

[1] Batina, J. T. Unsteady transonic algorithm improvements for realistic aircraft applications, Journal of Aircraft, 26, 2, 1989, pp. 131-139. C314, C315

[2] Engquist, B., and Osher, S. Stable and entropy satisfying approximations for transonic flow calculations, Mathematics of Computation, 34, 149, 1980, pp. 45-75. C317

[3] Gear, J. A., Ly, E., and Phillips, N. J. T. Time marching finite difference solution of the modified transonic small disturbance equation. In J. Noye, M. Teubner, and A. Gill, editors, Proceedings of the 8th Biennal Computational Techniques and Applications Conference (CTAC97), World Scientific, pp. 209-216. C312, C314, C315, C317

[4] Ly, E. Improved approximate factorisation algorithm for the steady subsonic and transonic flow over an aircraft wing. In Proceedings of the 21st Congress of the International Council of the Aeronautical Sciences (ICAS98), Melbourne, Australia, Sep. 1998, Paper A98-31699. C316

[5] Ly, E., and Gear, J. A. Time-linearized transonic computations including shock wave motion effects, Journal of Aircraft, 39, 6, 2002, pp. 964-972. C312, C314, C315 
[6] Ly, E., Gear, J. A., and Phillips, N. J. T. Improved approximate factorisation algorithm. In J. Noye, M. Teubner, and A. Gill, editors, Proceedings of the 8th Biennal Computational Techniques and Applications Conference (CTAC97), World Scientific, pp. 393-400. C316, C317

[7] Ly, E., and Nakamichi, J. Algorithm for calculating time-linearised transonic solutions including shock motion and shock-generated entropy and vorticity effects. In Proceedings of the Japan Society for Aeronautical and Space Sciences (JSASS) 16th International Sessions in 40th Aircraft Symposium Incorporating Japan-Korea Joint Symposium 2002, JSASS, Yokohama, Japan, Oct. 2002, pp. 117-120. C312, C314, C317

[8] Ly, E., and Nakamichi, J. Time-linearised transonic computations including entropy, vorticity and shock wave motion effects, The Aeronautical Journal, 107(1077), Nov. 2003, pp. 687-695. C312, C314, C317

[9] Phillips, N. J. T., Gear, J. A., and Ly, E. Type-dependent differencing schemes for transonic flow computation. In J. Noye, M. Teubner, and A. Gill, editors, Proceedings of the 8th Biennal Computational Techniques and Applications Conference (CTAC97), World Scientific, pp. 537-544. C317

[10] Tamayama, M., Saitoh, K., Matsushita, H., and Nakamichi, J. NAL SST arrow wing with oscillating flap. Verification and validation data for computational unsteady aerodynamics, Research and Technology Organization (RTO), RTO-TR-26, France, Oct. 2000, pp. 295-318. C311, C312, C320

[11] Tijdeman, H. Investigations of the transonic flow around oscillating airfoils. NLR, NLR-TR-77090U, The Netherlands, Oct. 1977. C314 
[12] Whitlow, W., Jr., Hafez, M. M., and Osher, S. J. An entropy correction method for unsteady full potential flows with strong shocks, Journal of Fluids and Structures, 1, 1987, pp. 401-414. C312, C317 